\title{
Correlations of the Number of Dewar Resonance Structures and Matching Polynomials for the Linear and Zigzag Polyacene Series ${ }^{\dagger}$
}

Jerry Ray Dias

\author{
Department of Chemistry, University of Missouri-Kansas City, Kansas City, MO 64110-2499, USA
}

RECEIVED JUNE 4, 2013; REVISED JULY 16, 2013; ACCEPTED JULY 16, 2013

\begin{abstract}
Linear and zigzag polyacene series have been the subject of numerous studies because of their contrasting electronic and stability characteristics. The correlation of the properties of these series is examined in regard to their number of Dewar resonance structures $(D S)$. Since resonance-theoretic methods require algorithms for determining the number of Dewar resonance structures $(D S)$, recursion equations for calculating $D S$ for these series are presented for the first time. Excellent correlations between DS and the absorption p-band, ionization energies, Hückel HOMO, Aihara's reduced HOMOLUMO gap, topological resonance energy (TRE), aromatic stabilization energy (ASE), and the Klein and Randić innate degree of freedom are presented and rationalized. (doi: 10.5562/cca2292)
\end{abstract}

Keywords: Dewar resonance structures, recursion equations, benzenoid hydrocarbons, polyacenes, resonance energies

\section{INTRODUCTION}

The matching polynomial (also, called acyclic polynomial) is useful for determining topological resonance energy $^{1,2}$ (TRE), the number of Kekulé resonance structures, and the number of Dewar resonance structures ${ }^{3}$ $(D S)$ of polycyclic conjugated hydrocarbons (PAH). Dewar structures consist of long bond (or excited) resonance structures that play a role in elucidating molecular reactivity, spin density, magnetic susceptibility, and electric conductance. ${ }^{4}$ While the coefficients of the characteristic polynomial can be computed by summing all the Sachs graphs, the coefficients of the matching polynomial are computed by summing all the Sachs graphs not having cyclic components. Thus, the matching polynomial is considered to be the reference polynomial of the hypothetical acyclic reference structure devoid of cyclic contributions against which the characteristic polynomial of a cyclic/polycyclic structure can be compared for purposes of determining resonance energy. The sum of the coefficients of the matching polynomial without regard to their negative signs is defined as the topological index $Z$ by Hosoya. ${ }^{5}$

Given this broad utility of the matching polynomial, we seek facile ways to determine it for molecular graphs corresponding to conjugated polycyclic hydrocarbons. In addition, the number of Dewar resonance structures is a measure of the triplet excited state energy for conjugated hydrocarbons and can be determined from the last two coefficients in the matching polynomial. It was shown by Linus Pauling that when essentially single bonds are present in a conjugated system, one must also employ Dewar resonance structures in addition to Kekule resonance structures to determine bond lengths of essentially single bonds. ${ }^{6}$ Subsequently, Seitz, Klein, Schmalz, and Garcia-Bach suggested that consideration of other structures besides Kekulé structures might be warranted in their study of electronic excitation, implying Dewar resonance structures. ${ }^{7}$ Thus, enumeration of Dewar resonance structures needs suitable computational algorithms.

Hosoya and coworkers showed ${ }^{8}$ that the infinite linear polyacene had a zero band gap whereas the infinite zigzag polyacene had a HMO band gap of HOMO$\mathrm{LUMO}=0.7639 \beta$. In regard to topological resonance energy (TRE), linear and zigzag polyacene isomers were compared to their cyclo-counterparts by Aihara. ${ }^{9}$ A comparison of the aromaticity of the linear and zigzag polyacenes was performed by the HOMA method. ${ }^{10} \mathrm{By}$ the topological measure of stability (TMS), the infinite zigzag polyacene is two times more stable than the infinite linear polyacene. ${ }^{11}$ As an example chrysene has a TMS $=0.7292$ and we calculated that triphenylene has a TMS $=0.7778$ indicating it is more stable than is chrysene. Linear polyacenes all have only one Clar sextet whereas the zizag polyacenes have an increasing

\footnotetext{
$\dagger$ Dedicated to Professor Douglas Jay Klein on the occasion of his $70^{\text {th }}$ birthday.

* Author to whom correspondence should be addressed. (E-mail: diasj@umkc.edu)
} 
number of Clar sextets as they increase in length leading to their increasing stability with increasing length. ${ }^{12}$ A para-delocalization index in six-membered rings as a criterion for aromaticity uses Dewar resonance structures restricted to hexagonal rings. It is illustrated with anthracene and phenanthrene in Scheme 2 in a paper by Solà and coworkers. ${ }^{13}$ Various properties for the linear and zigzag polyacenes with up to five rings were studied by Wiberg. ${ }^{14}$ In comparing linear acenes and phenacenes up to ten rings using natural orbital occupations, it was found that the linear acenes eventually became biradicals whereas phenacenes showed no indication of biradical character in this regime. ${ }^{15}$ Since linear (or just acenes) and zigzag (or phenacenes) polyacenes have been the subject of a number of comparative studies because of their contrasting stabilities, we will present our study of how changes in $D S$ correlates with various properties of these polycyclic aromatic hydrocarbons.

\section{RESULTS AND DISCUSSION}

The matching polynomial data of Hosoya and coworkers represents the most comprehensive source for all benzenoid hydrocarbons having up to seven rings. ${ }^{16}$ In addition, Hosoya and Ohkami presented an operator technique for deriving recursion formulas for both the characteristic and matching polynomials of selected polyacene series, including the linear and zigzag benzenoid hydrocarbons. ${ }^{17}$ A computer program to obtain the matching polynomial can be obtained from the work of Ramaraj and Balasubramanian. ${ }^{18}$

\section{Theory}

One can obtain the number of Dewar resonance structures for PAHs from the last two coefficients $\left[a_{N-2}{ }^{\text {ac }}\right.$ and $a_{N}{ }^{\text {ac }}$, i.e., the $\left(N-2^{\text {nd }}\right)$ and $N^{\text {th }}$ terms $]$ of the matching (acyclic) polynomials by using the following equation: ${ }^{3,19}$

$$
D S=\left|a_{N-2}^{\mathrm{ac}}+(N / 2) a_{N}^{\mathrm{ac}}\right|=\left|a_{N-2}{ }^{\mathrm{ac}}\right|-(N / 2) \boldsymbol{K}
$$

The number of Kekulé resonance structures $(\boldsymbol{K})$ is a measure of the singlet state energy of a molecule and the number of Dewar resonance structures is a measure of its triplet state energy. The closeness of energy of the triplet state to the singlet state is called diradical character of the molecule; singlet / triplet bistable molecules undergo facile thermal spin inversion between these two states and have a high percentage of diradical character, though a distinct valence-bond depiction can be written for them. We define diradical (or biradical) character by the following equation: ${ }^{19}$
BiRadChar $=\mathrm{BRC}=0.03 D S /(\boldsymbol{K}+0.03 D S)$

which is the weighted number of Dewar resonance structures divided by the total number of resonance structures of both types. ${ }^{19}$ Equation (2) is based on Pauling's weighting factor of 0.03 , i.e., a Dewar resonance structure carries only $3 \%$ the relative importance as does a Kekulé resonace structure. BRC is a measure of the triplet contribution to the closed shell ground state of a PAH.

TRE represents stabilization energy due to solely cyclic $\pi$-contribution which is in regard to $\pi$-binding energy of a graph-theoretically defined polyene reference; the first quantity is obtained from the characteristic polynomial and the second is obtained from the matching polynomial. ${ }^{1,2}$ The $\%$ TRE is defined as 100 times the TRE divided by the total binding energy of the polyene reference. TRE is an extensive property (naturally increases as molecular size increases) and \%TRE is an intensive property. ${ }^{20}$ Thus, the latter is used when comparing polycyclic aromatic molecules of different sizes. TRE and \%TRE are standard measures of aromatic stabilization energy $(A S E)$ and the reduced HOMO-LUMO gap is a measure of reactivity of the molecule. Microsoft Excel 2010 includes tools for plotting regression analysis per various functions and we selected the ones with the best correlation coefficients to give Figures 1-16.

\section{Number of Dewar Resonance Structures versus Various Properties}

Solution of the homogeneous Fibonacci recursion equation by standard methods gives the following equation for the number of Kekule resonance structures $\left(\boldsymbol{K}_{r}\right)$ of the phenacene series:

$$
\begin{aligned}
\boldsymbol{K}_{r}= & (3+\sqrt{5})(2 \sqrt{5})[(1+\sqrt{5}) / 2]^{r}+ \\
& (3-\sqrt{5})(2 \sqrt{5})[(1-\sqrt{5}) / 2]^{r}= \\
& 1.17082039325[1.61803398875]^{r}+ \\
& 0.17082039325[-0.61803398875]^{r}
\end{aligned}
$$

Note the last the term in equation (3), $0.17082039325[-0.61803398875]^{r}$, alternates in sign and becomes insignificant for $r>5$; it approaches a limit of zero as the number of rings $(r)$ increases infinitely. For example, for $r=13$ and 14, we get $\boldsymbol{K}_{13}=609.999672131-3.27869028686 \times 10^{-4}=$ 609.999344262 and $\boldsymbol{K}_{14}=987.000202635+$ $2.02634203587 \times 10^{-4}=987.000405269$; note that one rounds up to the nearest integer when the last term is 


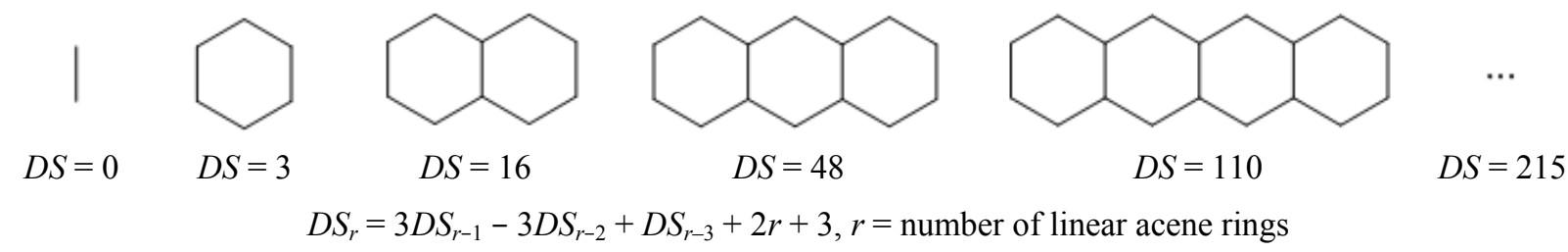<smiles>C1CCC2C(C1)CCC1CCCCC12</smiles>

phenanthrene $D S=47$<smiles>C1CCC2C(C1)CCC1CCCCC12</smiles>

chrysene<smiles>C1CCC2C(C1)CCC1C2CCC2C3CCCCC3CCC21</smiles>

picene<smiles>C1CCC2C(C1)CCC1C2CCC2C3CCC4CCCCC4C3CCC12</smiles>

fulminene

$D S=538$ $D S=1067$

$$
\begin{gathered}
D S=1067,2050,3845,7081,12853,23060,40979, \ldots ; \boldsymbol{K}_{r}=1.17082(1.618034)^{r} \\
D S_{r}=3 D S_{r-1}-2 D S_{r-2}-D S_{r-3}+D S_{r-4}+1.4223(1.618034)^{r}, r>5
\end{gathered}
$$

Figure 1. Recursion equations for the number of Dewar resonance structures for the linear and zigzag polyacenes. The linear polyacenes requires one to know the $D S$ values for the preceding three members of the series and for the zigzag polyacenes one needs to know the $D S$ values for the preceding four members.

negative and rounds down when it is positive. Clearly one would need a supercomputer to get the exact integer numbers of 610 and 987 , respectively, rather than a using a calculator to 10 significant figures. Because of this, we prefer to minimize the calculation effort without loss of accuracy by using the following truncated equation for $r>5$ :

$$
\boldsymbol{K}_{r}=1.17082[1.618034]^{r}
$$

Using equation (4) gives $\boldsymbol{K}_{13}=609.999522384$ and $\boldsymbol{K}_{14}=986.9999672$ which are rounded to the nearest integers of 610 and 987 , respectively.

Equations (5) and (6) (also Figure 1) present recursion equations that we derived for the linear acene and zigzag phenacene series, respectively:

$$
\begin{aligned}
D S_{r} & =3 D S_{r-1}-3 D S_{r-2}+D S_{r-3}+2 r+3, \\
r & =\text { number of linear acene rings }
\end{aligned}
$$

$$
\begin{gathered}
D S_{r}=3 D S_{r-1}-2 D S_{r-2}-D S_{r-3}+D S_{r-4}+ \\
1.4223[1.618034]^{r}, r>5 \text { for phenacene }
\end{gathered}
$$

For example, for $r=13$ and 14 equation (6) gives $D S_{13}=3 \times 23060-2 \times 12853-7081+3845+741.02$ $=40979.02$ and $D S_{14}=3 \times 40979-2 \times 23060-12853+$ $7081+1198.997=72243.997$, respectively; note that for $r=$ odd number one rounds down the resulting $D S$ value to the nearest integer and for $r=$ even number one rounds up to the nearest integer. In this way equation (6) always gives accurate $D S$ values for the phenacene series. From equations (5) and (6), it is evident that for the linear acenes one needs to know the $D S$ values for the preceding three members and for the zigzag phenacenes one needs the $D S$ values for the preceding

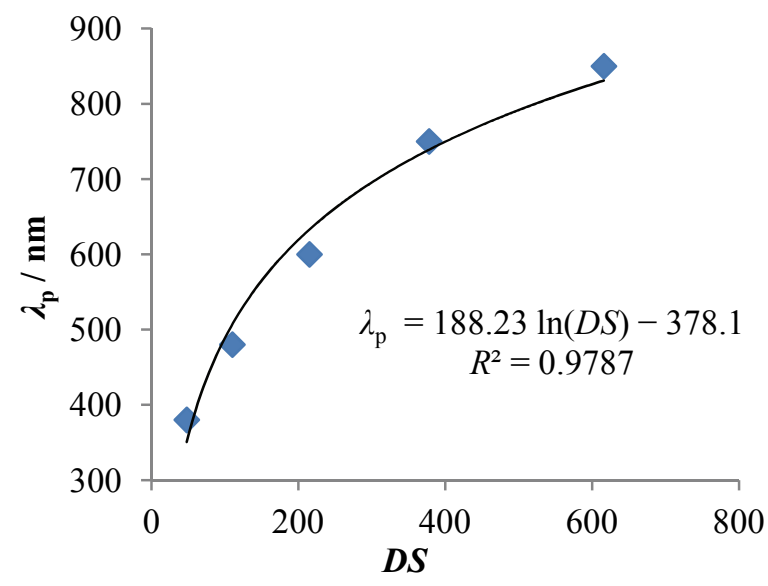

short axis polarization

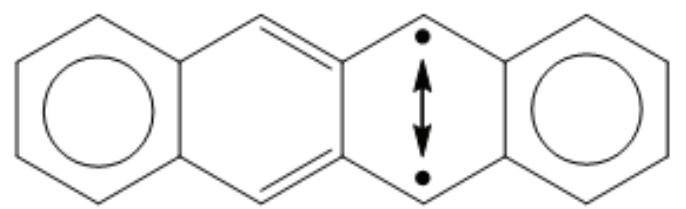

$D S=4$ for bisallyl substructure with two sextets

Figure 2. Number of Dewar resonance structures $(D S)$ versus $\lambda_{\mathrm{p}}(\mathrm{nm})$ for the linear polyacenes. 


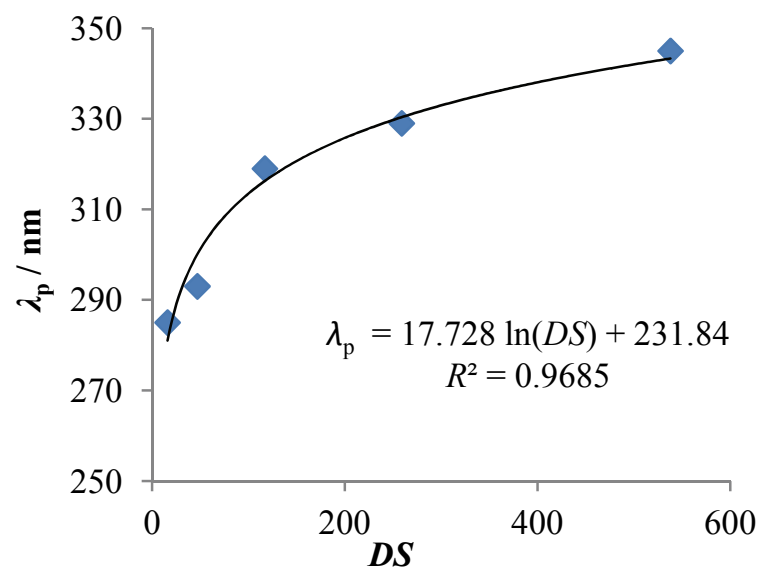

Figure 3. Number of Dewar resonance structures $(D S)$ versus $\lambda_{\mathrm{p}}(\mathrm{nm})$ for zigzag polyacenes.

four members. Except for the anthracene and phenanthrene pair of isomers, the $D S$ values for the phenacene isomers are larger than for the linear acenes. Most of the plots presented in this work start with anthracene and phenanthrene which can be regarded as the most straightforward representative members of the straight (linear) and kinked (zigzag) benzenoid polyacenes, respectively. Naphthalene could be regarded as the smallest member of both the linear and zigzag polyacene series, but as a rule the smallest member of a series is frequently the most anomalous.

Since the number of Dewar resonance structures is a measure of its triplet state energy and it is known that the electronic transition from the HOMO to the LUMO corresponds to the p-band in the UV spectrum, we plot $D S$ versus $\lambda_{\mathrm{p}}$ in Figures 2 and 3 for the linear acenes and the phenacenes, respectively. ${ }^{21}$ The $p$-band in the UV spectrum arises from polarization along the short axis of these molecules. Figure 2 shows an example with naphthacene which shows that many of the Dewar resonance structures are favored due generation of an

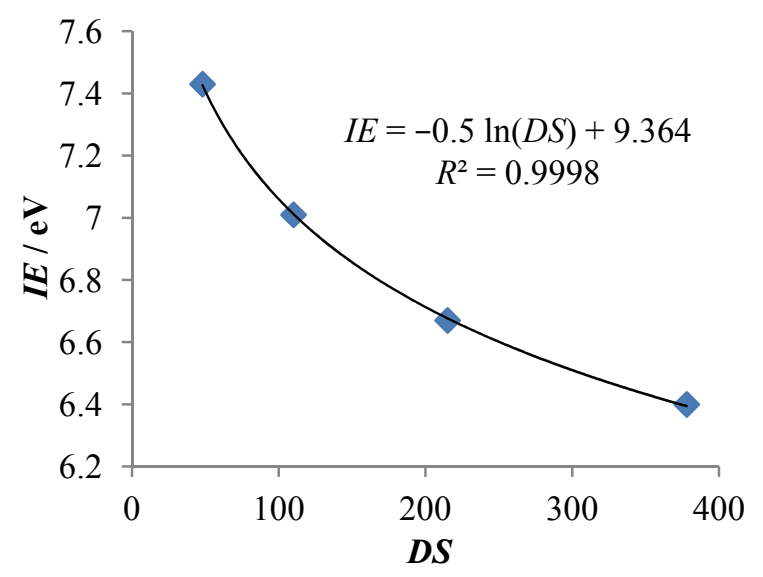

Figure 4. Number of Dewar resonance structures $(D S)$ versus ionization energies $(\mathrm{eV})$ of the linear polyacenes.

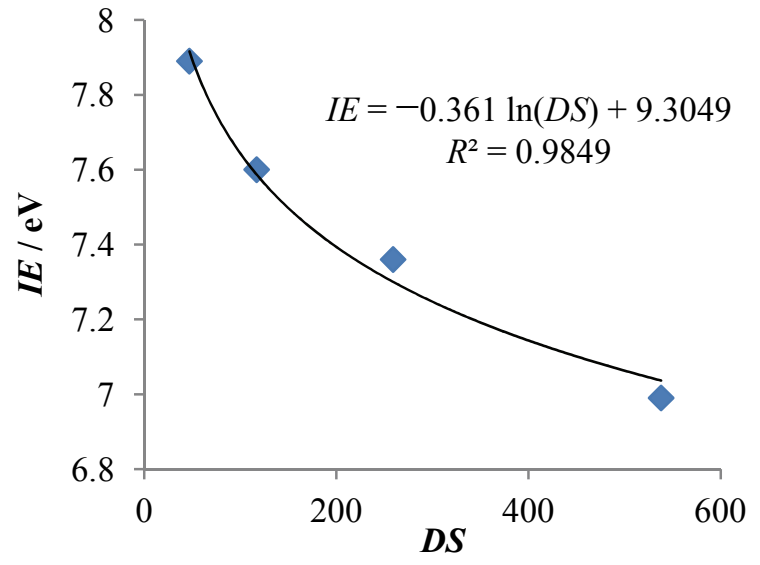

Figure 5. Number of Dewar resonance structures $(D S)$ versus ionization energies $(\mathrm{eV})$ of zigzag polyacenes.

additional sextet; in the electronic polarization of the zigzag phenacenes the number of sextets either remains unchanged or is reduced by one. The equation for the zigzag polyacenes in the Figure 3 gives $\lambda_{\mathrm{p}}=281 \mathrm{~nm}$ for naphthalene which is close to its experimental value of $\lambda_{\mathrm{p}}=285 \mathrm{~nm}$; the equation in Figure 2 for the linear polyacenes gives no agreement for naphthalene. Similar plots versus ionization energies (IEs) are given in Figures 4 and 5 . Other things being equal, the greater number of Dewar resonance structures, the easier it should be to remove an electron because long range spin pairing between two electrons is weaker than short range spin pairing between adjacent electrons. Both Figures 4 and 5 show that as the number of Dewar resonance structures increases in the given series, the $I E$ progressively decreases. For the linear polyacene series many of the Dewar resonance structures lead to increases in the number of sextets whereas for the zigzag polyacenes all the Dewar resonance structures lead to a decrease in the number of sextets. This explains why the $I E$ for the latter series is larger than for the former series. Wiberg found that the radical cations formed by ionization have the positive charge mainly located at the $\pi$-orbitals with the highest bond order. ${ }^{14}$ Phenacene isomers compared with the linear acenes generally have higher bond orders. The IEs were obtained from the National Standard Reference Data System. ${ }^{22}$ Using the equations in Figures 4-5 for the linear and zigzag polyacenes gives $I E=7.98$ and $8.30 \mathrm{eV}$, respectively, for naphthalene (experimental $I E=8.15 \mathrm{eV}$ ). Figures 2-5 all have logarithm relationships with the zigzag polyacenes having slightly less favorable correlations coefficients $\left(R^{2}\right)$.

The HOMO data in Figures 6-7 were obtained from two primary sources. ${ }^{9,23}$ The best correlation for the linear polyacenes was given by the power equation shown in Figure 6. As anticipated, the HOMO data in Figure 6 for the linear polyacenes approaches zero with increasing number of rings which coincides with 


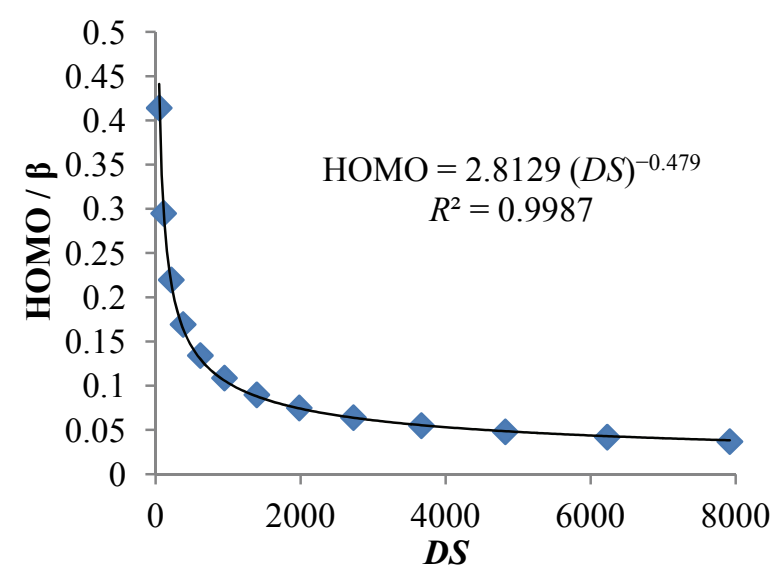

Figure 6. Number of Dewar resonance structures $(D S)$ versus $\mathrm{HOMO}(\beta)$ of linear polyacenes.

increasing $D S$ values. No equivalently good correlation for the zigzag polyacenes was found against their DS values; only a correlation of $R^{2}=0.85$ was obtained. However, the zigzag polyacenes were found to give the best correlation against their biradical character (BRC). ${ }^{19}$ In the plot of BRC versus HOMO, the zigzag phenacenes in Figure 7 appears to approach the Hosoya calculated limit HOMO value of $0.382 \beta$ as the number of rings increases; ${ }^{8}$ actually for $\mathrm{BRC}=1.0$ Figure 7 gives $\mathrm{HOMO}=0.36 \beta$ as a limit value. Alternatively, if we plot the HOMO data ${ }^{23}$ against the inverse of the number of phenacene rings $\left(r^{-1}\right)$ or against $D S^{-1}$, the extrapolated values of $0.355 \beta$ and $0.394 \beta$, respectively, are obtained. Finally, a plot of the HOMO data ${ }^{23}$ for linear polyacenes $(\mathrm{L})$ versus the HOMO for the zigzag polyacenes ( $\mathrm{Z}$ ) gives the extrapolated value of $\mathrm{HOMO}=0.392 \beta$ for the infinite zigzag polyacene at the $\mathrm{HOMO}=0$ value for the infinite linear polyacene $\left(\mathrm{Z}=0.1626 \mathrm{~L}^{2}+0.4344 \mathrm{~L}+0.3925\right)$.

Reduced HOMO-LUMO gap is defined as the HOMO-LUMO energy separation of a molecule

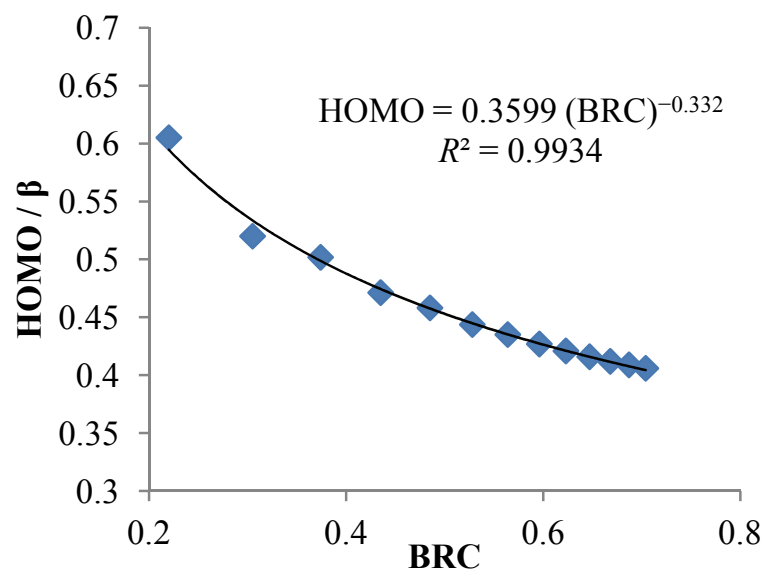

Figure 7. Biradical character (BRC) versus $\operatorname{HOMO}(\beta)$ for zigzag polyacenes.

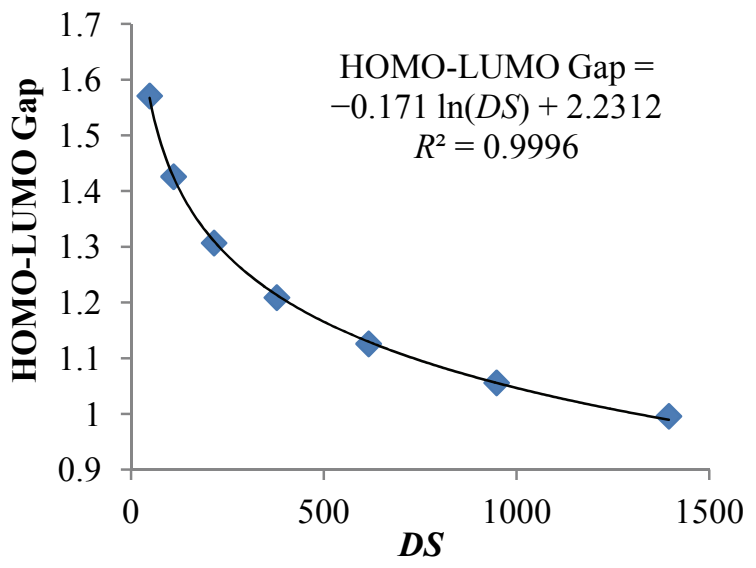

Figure 8. Number of Dewar resonance structures $(D S)$ versus reduced HOMO-LUMO gap for linear polyacenes.

(determined from the characteristic polynomial) divided by that of the hypothetical polyene reference which is devoid of cyclic contributions (determined from the matching polynomial) and is a measure of kinetic stability. ${ }^{24}$ The contrasting difference in reactivity (kinetic stability) of the linear acenes versus the phenacenes is emphasized by the plots (Figures 8 and 9) of the number of Dewar resonance structures versus the reduced HOMO-LUMO gap index of Aihara. ${ }^{24}$ If the reduced HOMO-LUMO gap index is $<1.3$, then the PAH is chemically very reactive. Starting with benzene all total resonant sextet benzenoids have reduced HOMO-LUMO gap index $>1.93$. This index shows that the reactivity of the linear acenes increases and for the phenacenes the reactivity decreases with increasing number of rings and $D S$ number.

By comparison, the TRE for the linear acenes and zigzag phenacenes ${ }^{9}$ both increases with increasing number of rings and the number of Dewar resonance structures as shown in Figures 10 and 11, respectively, but the latter increases faster presumably because all linear

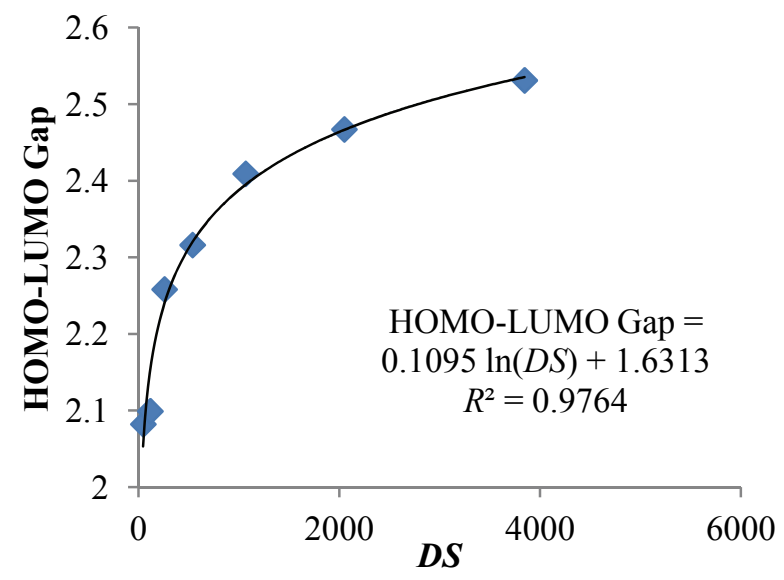

Figure 9. Number of Dewar resonance structures $(D S)$ versus reduced HOMO-LUMO gap for zigzag polyacenes. 


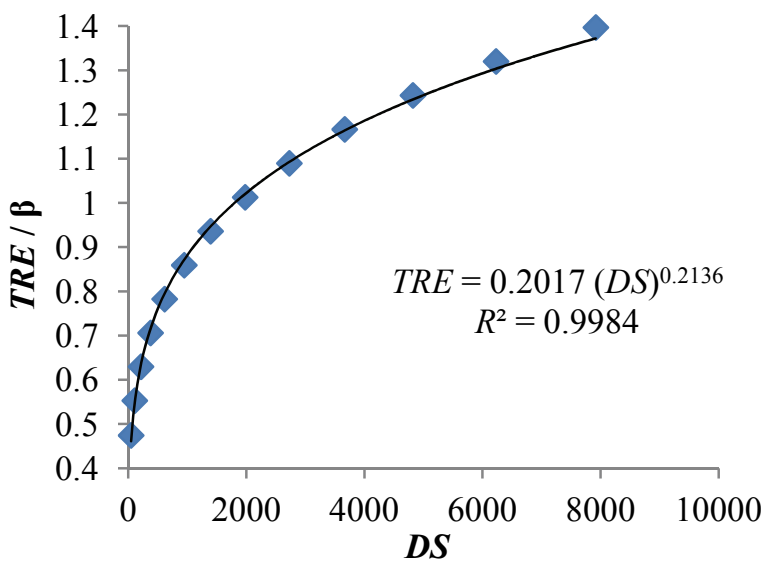

Figure 10. Number of Dewar resonance structures $(D S)$ versus $\operatorname{TRE}(\beta)$ for linear polyacenes.

acenes only have one resonant sextet while the number of resonant sextets progressively increases with every other added ring in the phenacenes. According to Aihara, ${ }^{24}$ reduced HOMO-LUMO gap index $<1.0$ indicates that the HOMO of the molecule contributes to the decrease in topological resonance energy; this occurs for nonacene which has a reduced HOMO-LUMO gap index of 0.996 and for longer linear acenes. The linear acenes in Figure 10 have a power plot equation and the phenacenes in Figure 11 have a logarithmic equation. For an additive property, one should use an additive invariant or the logarithm of a multiplicative invariant and $D S$ is a multiplicative invariant. This suggests that for the zigzag phenacenes their increasing number of sextets makes a stronger additive contribution to TRE compared to the linear acenes having only one sextet. In addition, for longer linear acenes their decreasing HOMO contributes to the decrease in their topological resonance energy. From another viewpoint, the vertical bonds on the end of a linear acene or between two adjacent rings has a low $\pi$-bond order of $\boldsymbol{K}^{-1}$, where

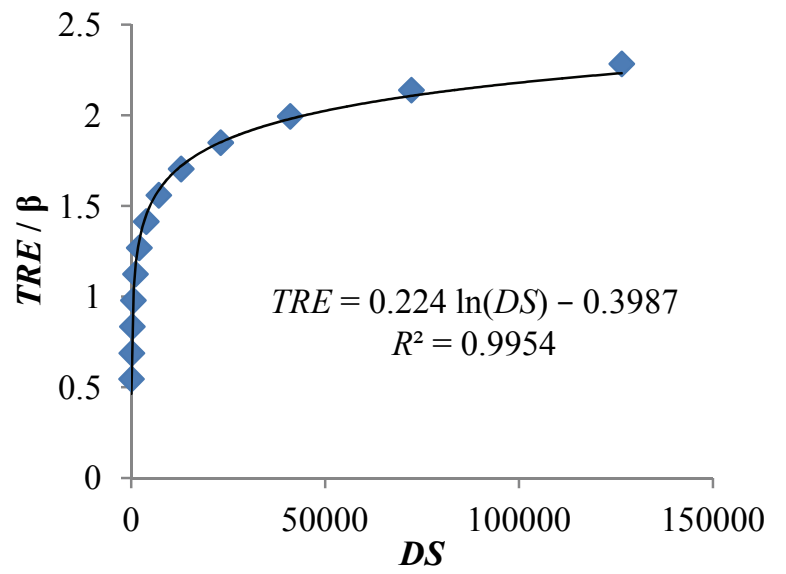

Figure 11. Number of Dewar resonance structures $(D S)$ versus TRE for zigzag polyacenes.

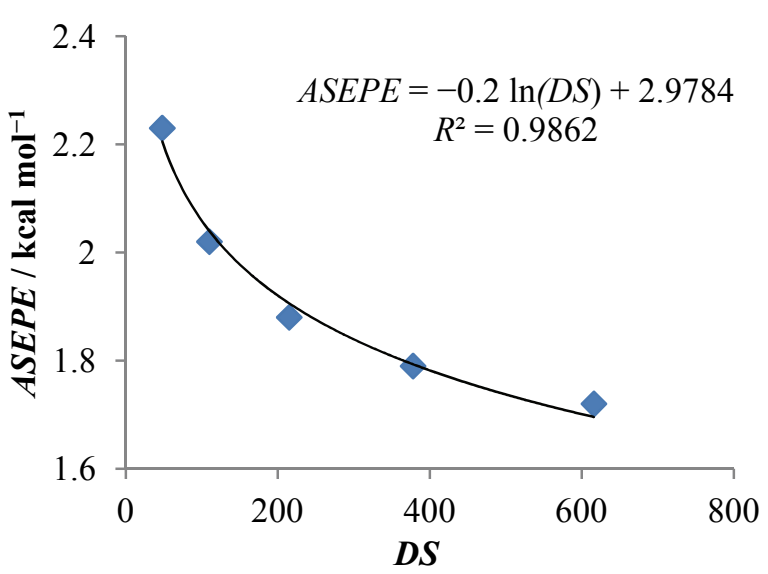

Figure 12. Number of Dewar resonance structures $(D S)$ versus ASEPE $\left(\mathrm{kcal} \mathrm{mol}^{-1}\right)$ for linear polyacenes.

$\boldsymbol{K}=r+1$, i.e., these vertical bond orders decrease approaching zero as the number of rings $(r)$ increase. Thus, adding another four-carbon diene fragment to this vertical end bond of a linear acene leads to relatively little additional stabilization. ${ }^{14}$ Whereas, the bond between two rings of a phenacene approaches a $\pi$-bond order of 0.61803 and addition of a four-carbon diene fragment to form another adjacent ring with this higher bond order will gain significantly more in electron delocalization and associated stabilization.

The plots in Figures 12-13 show the correlation between the $D S$ values and aromatic stabilization energy (ASE) per $\pi$-electron (i.e., $A S E P E$ ) for the linear and zigzag polyacenes, respectively. The $A S E$ values were computed using the Cohen and Benson group additivity method based on calorimetric data. ${ }^{25}$ These $A S E$ values were determined as the difference between the group additivity values for the aromatic system and those for the olefinic reference. ${ }^{10}$ Neither of these plots extrapolates well to naphthalene $(A S E P E=2.61)$ or benzene $(A S E P E=3.49)$.

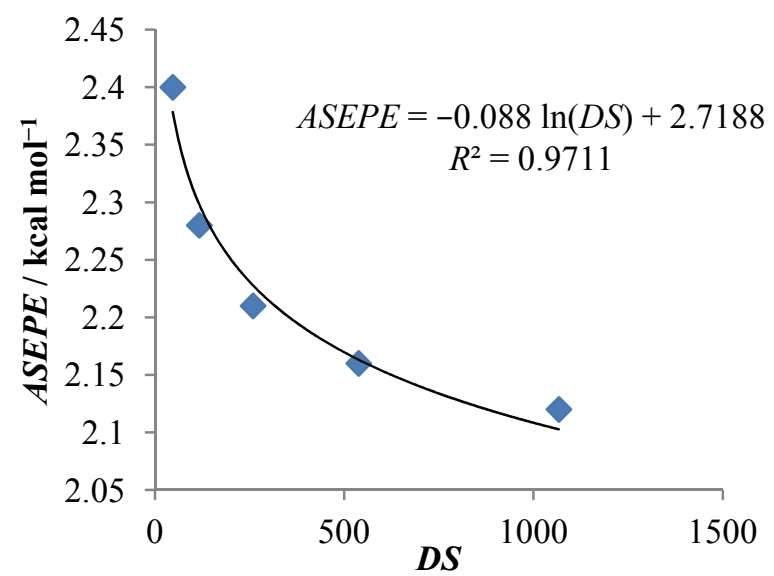

Figure 13. Number of Dewar resonance structures $(D S)$ versus $A S E\left(\mathrm{kcal} \mathrm{mol}^{-1}\right)$ per $\pi$-electron for zigzag polyacene. 


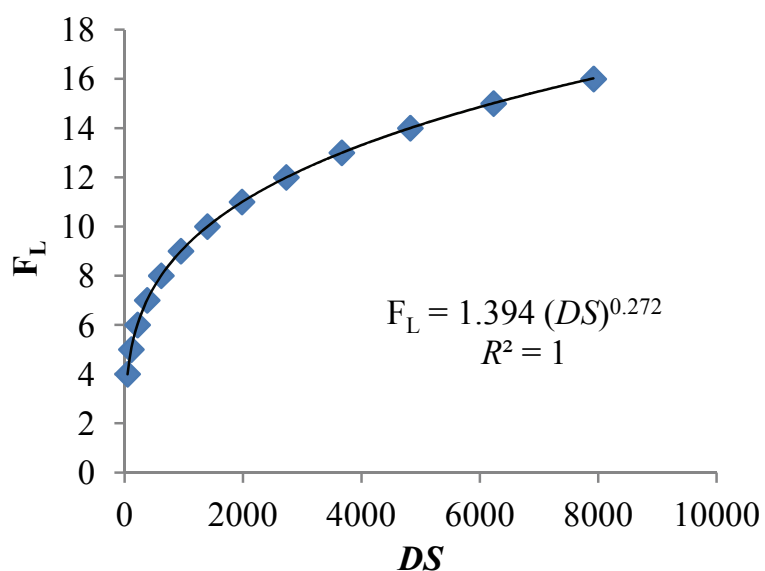

Figure 14. Number of Dewar resonance structures $(D S)$ versus innate degree of freedom $\left(\mathrm{F}_{\mathrm{L}}\right)$ for linear polyacenes.

Figures 14-15 give plots of DS versus innate degree of freedom $^{26}$ for the linear and zigzag polyacenes. For each Kekulé structure of a given molecular graph, there is assigned a number corresponding to the smallest number of placements of double bonds such that there is only one way that the placement of the remaining double bonds can occur. These numbers for each Kekulé structure depiction are summed to give what is called the degree of freedom of the parent graph. The degree of freedom for all the linear polyacenes is simply equal to the number of Kekule resonance structures $(\boldsymbol{K}=r+1)$. The degree of freedom all the zigzag polyacenes was determined by Klein and Randic to be an additive invariant that is equal to the number of disjoint conjugated 6-circuits (Figure 15). ${ }^{26}$ Their conclusion was that the degree of freedom of zigzag polyacenes measures graphical characteristics distinct from Clar's resonance-theoretic ideas. Comparing the highly correlative plots $\left(R^{2}=1\right)$ in Figures 14-15 shows distinctively different equations that are supportive of this conclusion.

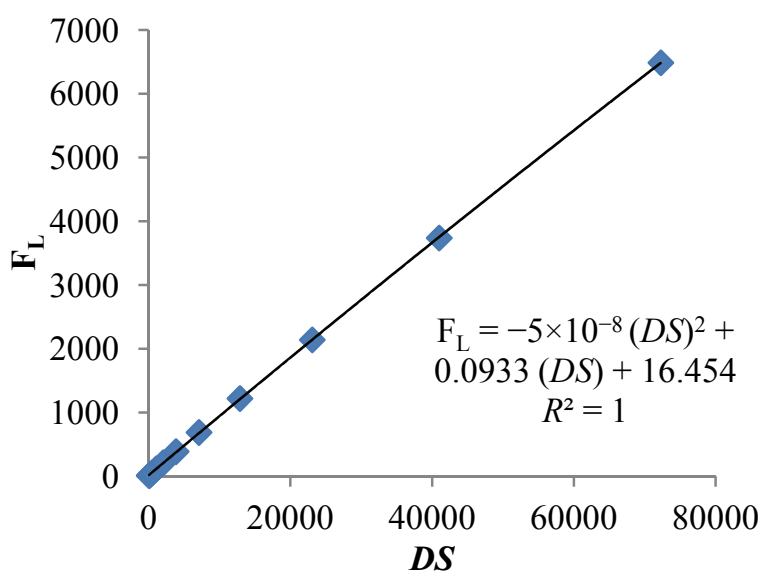

Figure 15. Number of Dewar resonance structures $(D S)$ versus innate degree of freedom $\left(\mathrm{F}_{\mathrm{L}}\right)$ for zigzag polyacenes.
In general, the Kekulé-structure-based model works well for small to moderate sized PAHs. Figure 16 gives a plot of $\left[(D S / \boldsymbol{K})_{\text {linear }} /(D S / \boldsymbol{K})_{\text {zigzag }}\right]$ against the number of rings which shows that the contribution of the triplet state (as measured by $D S$ ) relative to the ground state (as measured by $\boldsymbol{K}$ ) for the linear acenes is larger and grow faster than for the zigzag phenacenes. For example, $\left[(D S / \boldsymbol{K})_{\text {linear }} /(D S / \boldsymbol{K})_{\text {zigzag }}\right]=1.277$ for $r=3$ and $\left[(D S / \boldsymbol{K})_{\text {linear }} /(D S / \boldsymbol{K})_{\text {zigzag }}\right]=6.249$ for $r=15$. This explains that from the valence-bond point of view, the more reactive properties of the linear acenes. All linear acenes have two mobile Fries 6-circuits whereas all zigzag phenacenes can have Fries 6-circuits in every ring at once.

\section{Chrysene Anomaly}

Both the $a b$ initio calculated $\left(\mathrm{HF} / 6-31 \mathrm{G}^{* *}\right)$ and experimental $\Delta H_{f}^{0}$ values for chrysene suggest that it is more stable than its the total resonant sextet isomer, triphenylene. ${ }^{27-29}$ Herndon and coworkers attributed this anomaly as arising from a T-effect (opposing bay regions $\mathrm{S}_{3} \mathrm{~S}_{3}$ through a single hexagonal ring). Triphenylene has three $\mathrm{S}_{3} \mathrm{~S}_{3}$ pairs $=3 \mathrm{~T}$ and chrysene has none, thus leading to additional 'steric' destabilization of triphenylene. Perylene and benzo[e]pyrene both have one $\mathrm{S}_{3} \mathrm{~S}_{3}$ pair $=1 \mathrm{~T}$. In the determination of their topological measure of stability (TMS), Ciesielski and coworkers subtract out the total number of cis-butadiene units associated with bay regions in a given Kekulé structure whereas the Herndon and coworker's T-effect has the additional requirement that they be located pairwise on the same ring. Herndon and coworkers attribute this T-effect to additional 'steric' destabilization but an isolated $\mathrm{S}_{3}$ which corresponds to a bay region does not lead to any steric effect or destabilization. In this work, it is evident by inspection of the plots in Figures 3, 7, and 9 that chrysene deviates significantly from the curve. While deleting phenanthrene from Figure 3 does

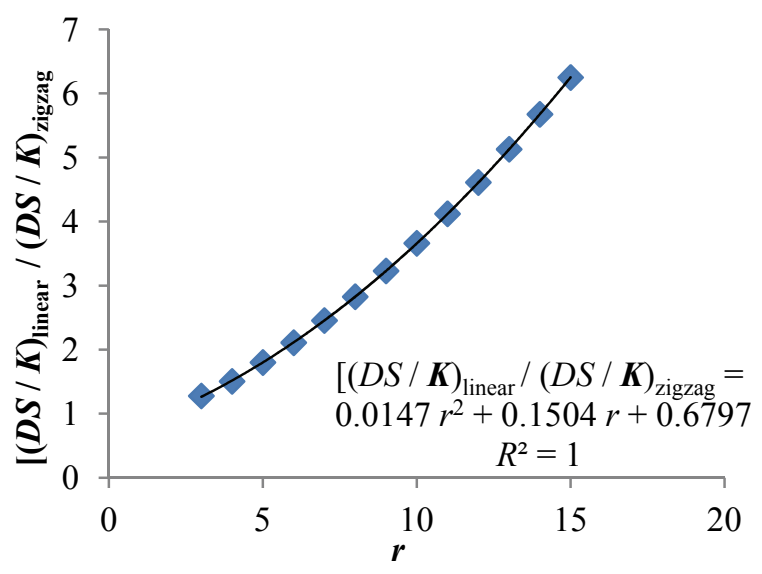

Figure 16. . Number of Rings $(r)$ versus $\left[(D S / \boldsymbol{K})_{\text {linear }} /\right.$ $\left.(D S / \boldsymbol{K})_{\text {zigzag }}\right]$. 
lead to some improvement, deleting only chrysene from all these plots leads to the best overall improvement in their correlations $\left(R^{2}\right)$. To explain this result of removing chrysene from the plots in Figures 3, 7, and 9, we note that HOMO is involved in both Figures 7 and 9. If we plot the HOMO versus $E_{\pi}(\beta)$ for the five $\mathrm{C}_{18} \mathrm{H}_{12}$ isomers of chrysene $\left(\mathrm{HOMO}=0.5201 \beta\right.$ and $E_{\pi}=$ $25.1922 \beta$ ), chrysene again deviates significantly from the curve. Plotting only the four isomers of chrysene gave the equation $E_{\pi}=3.1455(\mathrm{HOMO})^{3}$ $5.3642(\mathrm{HOMO})^{2}+3.755(\mathrm{HOMO})+24.209\left(R^{2}=1\right)$; solution of this equation using HOMO $=0.5201 \beta$ gives $E_{\pi}=25.1535 \beta$ for chrysene. Alternatively, the inverse equation $\mathrm{HOMO}=-4.6483\left(E_{\pi}\right)^{3}+351.25\left(E_{\pi}\right)^{2}-$ $8846.1\left(E_{\pi}\right)+74251.284\left(R^{2}=1\right)$ gives HOMO $=0.5716$ $\beta$ for chrysene using $E_{\pi}=25.1922 \beta$. Thus, our regression analysis shows that the HOMO and $E_{\pi}$ of chrysene also appears to be anomalous just like its enthalpy of formation. Among other thermochemical perspectives, Slayden and Liebman discussed the chrysene anomaly without any definitive resolution except that the sole experimental measurement of chrysene is questionable. $^{30}$

\section{CONCLUSION}

Kekulé based resonance theory works well for small to moderate sized benzenoids because a Dewar resonance structure is only $3 \%$ as important as a Kekulé resonance structure $^{3,6}$ and in many extended series both change in parallel to each other, though at different rates. However, improvement of the Kekule based resonance theory can be achieved by augmenting it with the number of Dewar resonance structures, especially for systems with essentially fixed single or double bonds and having one of very few Kekulé structures. ${ }^{31}$ In certain circumstances, knowing a select number of Dewar resonance structures is important in the calculation of special properties, like electric conductance and paradelocalization index. ${ }^{4,13}$ Graphene and related $\pi$ systems have been touted as having electro-optical properties with the potential to revolutionize engineering, computing and electronics. For example, polycyclic aromatic hydrocarbons with open-shell ground state (singlet or triplet biradical) have the potential to be nanoscale replacements for electronically bistable devices already in use for information storage. ${ }^{32}$ Pentacene is currently being used in field effect transistors.

Acknowledgements. This work was supported in part by a grant from by the UM Board of Curators (K0906077).

\section{REFERENCES}

1. J. Aihara, J. Am. Chem. Soc. 98 (1976) 2750-2758.

2. I. Gutman, M. Milun, and N. Trinajstic, J. Am. Chem. Soc. 99 (1977) 1692-1704.

3. J. R. Dias, J. Phys. Chem. A 115 (2011) 13619-13627.

4. T. Morikawa, S. Narita, and D. J. Klein, Chem. Phys. Lett. 402 (2005) 554-558.

5. (a) H. Hosoya, Bull. Chem. Soc. Jpn. 44 (1971) 2332-2339; (b) H. Hosoya, Internet Electronic J. Molec. Design 1 (2002) 428-442.

6. L. Pauling,. Acta Cryst. B 36 (1980) 1898-1901.

7. W. A. Seitz, D. J. Klein, T. G. Schmalz, and M. A. Garcia-Bach, Chem. Phys. Lett. 115 (1985) 139-143.

8. H. Hosoya, M. Aida, R. Kumagai, and K. Watanabe, J. Comput. Chem. 8 (1987) 358-366.

9. J. Aihara, J. Chem. Soc. Perkin Trans. 2 (1994) 971-974.

10. M. K. Cyranski, B. T. Stepien, and T. M. Krygowski, Tetrahedron 56 (2000) 9663-9667.

11. A. Ciesielski, T.M. Krygowski, and M. K. Cyranski, J. Chem. Inf. Model. 48 (2008) 1358-1366.

12. A. Mistra, D. J. Klein, and T. Morikawa, J. Phys. Chem. A 113 (2009) 1151-1158.

13. J. Poater, X. Fadera, M. Duran, and M. Solà, Chem. Eur. J. 9 (2003) 400-406.

14. K. B. Wiberg, J. Org. Chem. 62 (1997) 5720-5727.

15. F. Plasser, H. Pasalic, M. H. Gerzabek, F. Libsch, R. Reiter, J. Burgdorfer, T. Muller, R. Shepard, and H. Lischka, Angew. Chem. Int. Ed. 52 (2013) 2581-2584.

16. (a) T. Yamaguchi, M. Suzuki, and H. Hosoya, Natural Sci. Report, Ochanomizu Univ. 26 (1975) 39-60; (b) H. Hosoya, A. Uchiyama, M. Kadota, K. Chida, M. Aida, and T. Yamaguchi Natural Sci. Report, Ochanomizu Univ. 37 (1986) 133-168.

17. H. Hosoya and N. Ohkami, J. Comput. Chem. 4 (1983) 585-593.

18. R. Ramaraj and K. Balasubramanian, J. Comput. Chem. 6 (1985) 122-141.

19. J. R. Dias, J. Phys. Chem. A 117 (2013) 4716-4725.

20. M. Makino and J. Aihara, Phys. Chem. Chem. Phys. 10 (2008) 591-599.

21. R Rieger and K. Müllen, J. Phys. Org. Chem. 23 (2010) 315-325.

22. R. D. Levin and S. G. Lias, S.G. NSRDS-NBS 71 (1982) 86-120.

23. J. R. Dias, Handbook of Polycyclic Hydrocarbons, Part A; Elsevier: Amsterdam, NY, 1987, p. 128.

24. J. Aihara, J. Phys. Chem. A 103 (1999) 7487-7495.

25. N. Cohen and S. W. Benson, Chem. Rev. 93 (1993) 2419-2436.

26. D. J. Klein and M. Randić, J. Comput. Chem. 8 (1987) 516-521.

27. (a) W. C. Herndon, P. C. Nowak, D. A. Connor, and P. Lin, J. Am. Chem. Soc. 114 (1992) 41-47; (b) W. C. Herndon, J. Org. Chem. 63 (1998) 7445-7448.

28. T. M. Krygowski and M. K. Cyranski, Chem. Rev. 101 (2001) 1385-1419.

29. J. Yu, R. Sumathi, and W. H. Green Jr., J. Am. Chem. Soc. 126 (2004) 12685-12700.

30. S. W. Slayden and J. F. Liebman, Chem. Rev. 101 (2001) 1541-1566.

31. J. R. Dias, Polycycl. Aromat. Comp. 33 (2013) 138-150.

32. J. R. Dias, Molec. Phys. 11 (2013) 735-751. 